\title{
Avaliação da estrutura fitossociológica de um fragmento florestal no Município de Paragominas-PA, Brasil
}

\author{
Evaluation of the phytosociological structure of a forest fragment in the Municipality of \\ Paragominas-PA, Brazil \\ Evaluación de la estructura fitosociológica de un fragmento de bosque en el Municipio de \\ Paragominas-PA, Brasil
}

Recebido: 18/06/2021 | Revisado: 28/06/2021 | Aceito: 22/07/2021 | Publicado: 29/07/2021

Ana Cláudia Vale do Nascimento
ORCID: https://orcid.org/0000-0002-6301-5934
Bosque Rodrigues Alves, Brasil
E-mail: anacvn @ gmail.com
Paulo Luiz Contente de Barros
ORCID: https://orcid.org/0000-0003-2006-838X
Universidade Federal Rural da Amazônia, Brasil
E-mail: contente.barros@ hotmail.com
Gracialda Costa Ferreira
ORCID: https://orcid.org/0000-0002-4018-9624
Universidade Federal Rural da Amazônia, Brasil
E-mail: gracialda.ferreira@ @ufra.edu.br
Jéssica Costa dos Santos
ORCID: https://orcid.org/0000-0002-4424-1821
Universidade de São Paulo, Brasil
E-mail: jessicasantos.ufra@ gmail.com
Francimary da Silva Carneiro
ORCID: https://orcid.org/0000-0002-1693-8779
Universidade Federal Rural da Amazônia, Brasil
E-mail: francimarycarneiro@ gmail.com

\section{Resumo}

O objetivo do estudo foi analisar a estrutura fitossociológica do fragmento de floresta primária antropizada, no município de Paragominas no Estado do Pará. O nome vernacular, a circunferência a altura de 1,3 m do solo - CAP $\geq$ $31,5 \mathrm{~cm}$ (DAP $\geq 10 \mathrm{~cm}$ ), e a altura $(\mathrm{H})$ de todos os indivíduos das espécies arbóreas foram obtidos em 12 unidades amostrais de $20 \mathrm{~m}$ x $50 \mathrm{~m}$, distribuídas aleatoriamente na área. A suficiência amostral foi avaliada pelo ajuste da curva espécie-área gerada entre a área das parcelas e o número de espécies novas. Pela amostragem foram registrados 320 indivíduos classificados em 29 famílias botânicas, 44 gêneros e 46 espécies definidas e 4 morfoespécies. Em relação à estrutura horizontal, a espécies com maior abundância foi Inga alba (9,7\%). Eschweilera coriacea destacou-se com $13,31 \%$ de dominância na área estudada, o que contribuiu para que se tornasse a espécie com maior valor de importância da área com 8,59 \% de participação. Com uma ocorrência média de 41 espécies /ha de composição florística, a área do fragmento florestal apresentou uma diversidade de espécies expressa pelo índice de ShannonWeaner igual a 3,29, considerada baixa, quando comparada com florestas da mesma região. Quanto a classificação das espécies em grupos ecológicos, as espécies pioneiras e secundárias tardias constituem grupos equivalentes a $30 \%$ da composição florística, caracterizando que sob o dossel da floresta predominam as tolerantes à sombra e, as espécies pertencentes ao grupo secundárias iniciais, contribuíram com $28 \%$, evidenciando avançado estágio de sucessão ecológica. Por outo lado, a ocorrência de $30 \%$ de secundárias tardias, bem como de pioneiras podem ser explicadas pela exploração madeireira ocorrida na área do fragmento. Com relação ao tipo de vida no fragmento florestal $90 \%$ das espécies são árvores, $8 \%$ arbustos, e $2 \%$ palmeiras, com forte predominância de árvores.

Palavras-chave: Amazônia; Análise florística; Floresta secundária.

\begin{abstract}
The study objective was to analyze the vegetation structure of the anthropic primary forest fragment in the municipality of Paragominas in Para. The vernacular name, the circumference of the height of $1.3 \mathrm{~m}$ above the ground - CAP $\geq 31.5 \mathrm{~cm}(\mathrm{DBH} \geq 10 \mathrm{~cm})$ and height $(\mathrm{H})$ of all individuals of tree species were collected in 12 sampling units of $20 \mathrm{mx} 50 \mathrm{~m}$, randomly distributed in the area. The sample sufficiency was evaluated by adjusting the generated species-area curve between the area of the parcels and the number of new species. The sample was recorded 320 individuals classified into 29 botanical families, 44 genera and 46 species defined and 4 morphospecies.
\end{abstract}


To the horizontal structure, the species with the highest abundance was Inga alba (9.7\%). Eschweilera coriacea stood out with $13.31 \%$ of dominance in the study area, which helped it to become the species with the highest importance value of the area with $8.59 \%$ market share. With an average occurrence of 41 species / ha of floristic composition, the area of forest fragment showed a diversity of species expressed by the Shannon-Weaner index of 3.29, considered low compared with forests in the same region. As for classification of species in ecological groups, the late pioneer and secondary species are groups equivalent to $30 \%$ of the floristic composition, characterizing that under the forest canopy dominated by shade-tolerant and species belonging to the early secondary group, contributed $28 \%$, showing an advanced stage of ecological succession. By outo hand, the occurrence of $30 \%$ of late secondary and pioneer can be explained by logging occurred in the fragment area. Regarding the type of life in the forest fragment $90 \%$ of the species are trees, bushes $8 \%$ and $2 \%$ palm trees, with strong predominance of trees.

Keywords: Amazon; Floristic analysis; Secondary forest.

\section{Resumen}

El objetivo del estudio fue analizar la estructura fitosociológica del fragmento de bosque primario antropizado, en el municipio de Paragominas, Estado de Pará. El nombre vernáculo, la circunferencia a una altura de $1.3 \mathrm{~m}$ del suelo $\mathrm{CAP} \geq 31.5 \mathrm{~cm}(\mathrm{DAP} \geq 10 \mathrm{~cm}$ ), y la altura $(\mathrm{H})$ de todos los individuos de la especie arbórea se obtuvieron en 12 unidades de muestreo de $20 \mathrm{mx} 50 \mathrm{~m}$, distribuidas aleatoriamente en el área. La suficiencia del muestreo se evaluó ajustando la curva especie-área generada entre el área de las parcelas y el número de nuevas especies. Por muestreo se registraron 320 individuos clasificados en 29 familias botánicas, 44 géneros y 46 especies definidas y 4 morfoespecies. En relación a la estructura horizontal, la especie con mayor abundancia fue Inga alba $(9,7 \%)$. Se destacó Eschweilera coriacea con un 13,31\% de dominancia en el área estudiada, lo que contribuyó a que se convirtiera en la especie con mayor valor de importancia en el área con un 8,59\% de participación. Con una ocurrencia promedio de 41 especies / ha de composición florística, el área de fragmentos de bosque presentó una diversidad de especies expresada por el índice de Shannon-Weaner igual a 3.29, considerada baja en comparación con los bosques de la misma región. En cuanto a la clasificación de especies en grupos ecológicos, las especies pioneras y secundarias tardías constituyen grupos equivalentes al 30\% de la composición florística, caracterizándose que bajo el dosel del bosque predominan las tolerantes a la sombra y las especies pertenecientes al grupo secundario inicial contribuyeron con $28 \%$, mostrando una etapa avanzada de sucesión ecológica. Por otro lado, la ocurrencia del $30 \%$ de secundarias tardías, así como pioneras, puede explicarse por la tala que se realizó en la zona del fragmento. En cuanto al tipo de vida en el fragmento de bosque, el $90 \%$ de las especies son árboles, el $8 \%$ arbustos y el $2 \%$ palmeras, con fuerte predominio de árboles.

Palabras clave: Amazonas; Análisis florístico; Bosque secundario.

\section{Introdução}

As florestas tropicais brasileiras são formadas por um alto grau de diversidade de espécies arbóreas (Martins, 2012). A falta de conhecimento acerca da maioria dessas espécies nativas que compõem esse mosaico florestal, dificulta sua melhor interpretação em estudos nos diversos âmbitos da pesquisa (De Andrade Lima, 2012). Pesquisadores defendem a aplicação desses conhecimentos no planejamento das ações de gestão ambiental como no manejo florestal e na recuperação de área degradadas (Chaves et al., 2013).

Para Lima et al. (2012), o emprego de critérios florísticos e fitossociológicos aumentam o conhecimento dos importantes ecossistemas que compõem fragmentos florestais, e contribuem para o estabelecimento de processos chaves na manutenção de florestas, bem como auxiliam na elaboração de práticas ecológicas eficientes.

A partir de análises fitossociológicas e da composição florística da vegetação é possível definir um ecossistema de referência, e este servirá de modelo para o processo de restauração de áreas degradadas (Ser, 2004). Também, os conhecimentos dessas análises irão auxiliar no processo de avaliação da restauração dos ecossistemas degradados (Ser, 2004), bem como, por meio da caracterização de estágios de sucessão florestal, através dos parâmetros fitossociológicos como a abundância, a dominância, a frequência e o valor de importância (Hacck et al., 2005).

Esses parâmetros são bastante utilizados, sendo importante citar os trabalhos de (Lamprecht, 1964; Finol, 1975; Barros 1986), os quais descrevem a abundância absoluta (Aa) como sendo o número de indivíduos da espécie por unidade de área (ha), já a abundância relativa (Ar) a percentagem referente a Aa da espécie em relação ao número total de indivíduos por hectare de todas as espécies presentes na amostra. A dominância absoluta (Da) é a área basal da espécie, enquanto que a 
dominância relativa (Dr) é obtida através da (Da) de cada espécie em relação a área basal total (G) de todas as espécies. A frequência absoluta $(\mathrm{Fa})$ é expressa pela relação entre o número de unidades de amostra em que ocorreu a espécie pelo número total de amostras estabelecidas, e a frequência relativa $(\mathrm{Fr})$ é determinada através da relação entre a frequências absolutas da espécie pela soma das frequências absolutas de todas as espécies. Com esses parâmetros conhecidos é possível avaliar a capacidade produtiva da floresta e o potencial de cada espécie dentro da composição florística da vegetação do (Hosokawa, 2008).

Assim, o valor de importância é estimado por espécie, pela soma dos valores relativos da abundância, dominância e frequência, e demonstra a importância ecológica da espécie na comunidade.

$\mathrm{Na}$ análise da estrutura fitossociológica de uma comunidade, como o caso de um fragmento florestal, também, podem ser incluídas as estimativas de índices de similaridade, de diversidade, de agregação e associação de espécies (Souza E Soares, 2013; Barros, 1986).

Em geral, o Índice de Shannon-Wiener, é um dos mais utilizados para a obtenção da quantificação da biodiversidade em ecossistemas vegetais. Possibilitando a comparação em diferentes tipos de vegetação (Souza E Soares, 2013). Segundo Felfili e Rezende (2003), os valores deste índice, geralmente situam-se entre 1,3 e 3,5, podendo exceder 4,0 e alcançar em torno de 4,5 em florestais tropicais.

Almeida, et al. (2015) citam que as florestas são formadas por meio de sucessão, considerando que espécies são melhor adaptadas às condições de maior luminosidade colonizando rapidamente as áreas abertas propiciando condições favoráveis para espécies mais tardias. Assim, a classificação das espécies em grupos ecológicos, são informações importantes nos trabalhos de recuperação de ambientes alterados.

Desta forma, o objetivo do presente estudo foi avaliar a estrutura fitossociológica do fragmento de floresta primária antropizada, no município de Paragominas no Estado do Pará.

\section{Metodologia}

Este estudo foi realizado no Platô Miltônia III, local de exploração de bauxita, da empresa Mineração Paragominas S.

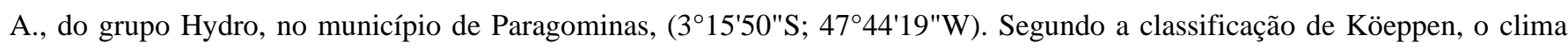
predominante na região é quente e úmido do tipo Awi, isto é, tropical chuvoso, com significativo período de estiagem. A temperatura média mínima anual é de $26,3^{\circ}$, com umidade relativa do ar elevada, apresentando um valor médio de $81 \%$ com precipitação pluviométrica média anual de Paragominas é de 1.742,9 mm (Bastos et al., 2005).

A obtenção dos dados de campo se deu através de um sistema de amostragem onde o método utilizado foi o de área fixa, com unidades de amostra de forma retangular com dimensões de $20 \mathrm{~m}$ x $50 \mathrm{~m}$ e, o processo amostral foi o aleatório com a distribuição de 12 unidades de amostra distribuídas na área do fragmento estudado onde foram registrados o nome vernacular, a circunferência a altura de $1,3 \mathrm{~m}$ do solo $-\mathrm{CAP} \geq 31,5 \mathrm{~cm}$ e a altura $(\mathrm{H})$ de todos os indivíduos das espécies arbóreas.

A suficiência amostral foi determinada pelo ponto de inflexão da curva espécie-área gerada entre o número de parcelas e o número de espécies, a qual avalia a amostragem mínima a ser realizada em comunidades vegetais. Para isso, foram testadas as equações linear e potencial.

Para a identificação científica das espécies foram obtidas amostras botânicas em campo, e posteriormente enviadas para o herbário da Embrapa Amazônia Oriental, PA. As nomenclaturas dos nomes científicos foram confirmadas utilizando o banco de dados eletrônico da Flora do Brasil, e dessa forma o sistema de classificação botânica adotado foi o APG III (2009).

Após a identificação botânica das espécies, estas foram classificadas quanto à forma de vida: arbóreas, arbustivas, lianas, ervas e palmeiras de acordo com Ribeiro et al. (1999) e qualificadas em grupos ecológicos: pioneiras, secundárias 
iniciais e secundárias tardia de acordo com (Gandolfi et al., 1995).

A estrutura fitossociológica da área foi determinada pelos seguintes parâmetros:

Abundância absoluta e relativa, Dominância absoluta e relativa, Frequência absoluta e relativa e o Índice de Valor de Importância, além do índice de diversidade de Shannon, quais são amplamente usados na literatura que tratam de estudos de vegetação (Lamprecht et al., 1964; Finol, 1975; Brower \& Zar, 1977; Barros, 1986; Coelho et al., 2003; Felfili \& Resende, 2003; Moro \& Martins, 2011; Longhi et al., 1999).

A suficiência amostral foi testada pelo ajuste da curva espécie-área (Figura 1), ajustando-se dois modelos matemáticos: (a) modelo potencial, $S=b_{0} x A^{b_{1}}$, linearizado com logaritmo decimal, $\log S=\log b_{0}+b_{1} \log A$ e (b) o modelo linear, $S=b_{0}+b_{1} \ln A$, onde $b_{0}$ e $b_{1}$, os coeficientes da equação, $\mathrm{A}=$ área amostrada, log $=$ logaritmo decimal e $\ln =\operatorname{logaritmo}$ natural.

\section{Resultados e Discussão}

Analisando a qualidade do ajuste das equações de regressão testadas (tabela 1), observa-se que ambas as equações apresentaram bons ajustes, com o teste $\mathrm{F}$, altamente significativo e com os Coeficientes de determinação $\left(\mathrm{r}^{2}\right)$ superiores a $90 \%$. Entretanto, com relação ao Coeficiente de Variação (CV \%), a equação potencial linearizada, (log S = -0,92604 + 0,64898* $\log$ A), apresentou o menor valor ( $\mathrm{CV} \%=1,54)$, quando comparado com o mesmo critério da função linear, $\mathrm{S}=-117,79+17,714 * \ln$ $\mathrm{A}$, que apresentou um CV \% $=11,25 \%$ muito superior.

Assim, com base nesse critério de qualidade de ajuste, modelo matemático que melhor ajustou a função espécie-área foi o modelo potencial, resultando na equação linearizada, $S=10^{[-0,92604+0,64898 \times \log A)}, \operatorname{com} r^{2}=98,8 \% \in C V \%=1,54 \%$.

A partir da curva ajustada, observa-se que mesmo não havendo uma estabilização perfeita da curva espécie-área, a amostra utilizada de $\left(12000 \mathrm{~m}^{2}\right)$, tende a uma estabilização, o que leva a considerar que a amostragem não foi suficiente para cobrir completamente a riqueza florística da área do fragmento (Figura 1 e Tabela 1), para representar perfeitamente a composição florística do fragmento.

Figura 1. Curva espécie-área ajustada pelo modelo potencial. Mineração Paragominas S.A, Paragominas-PA.

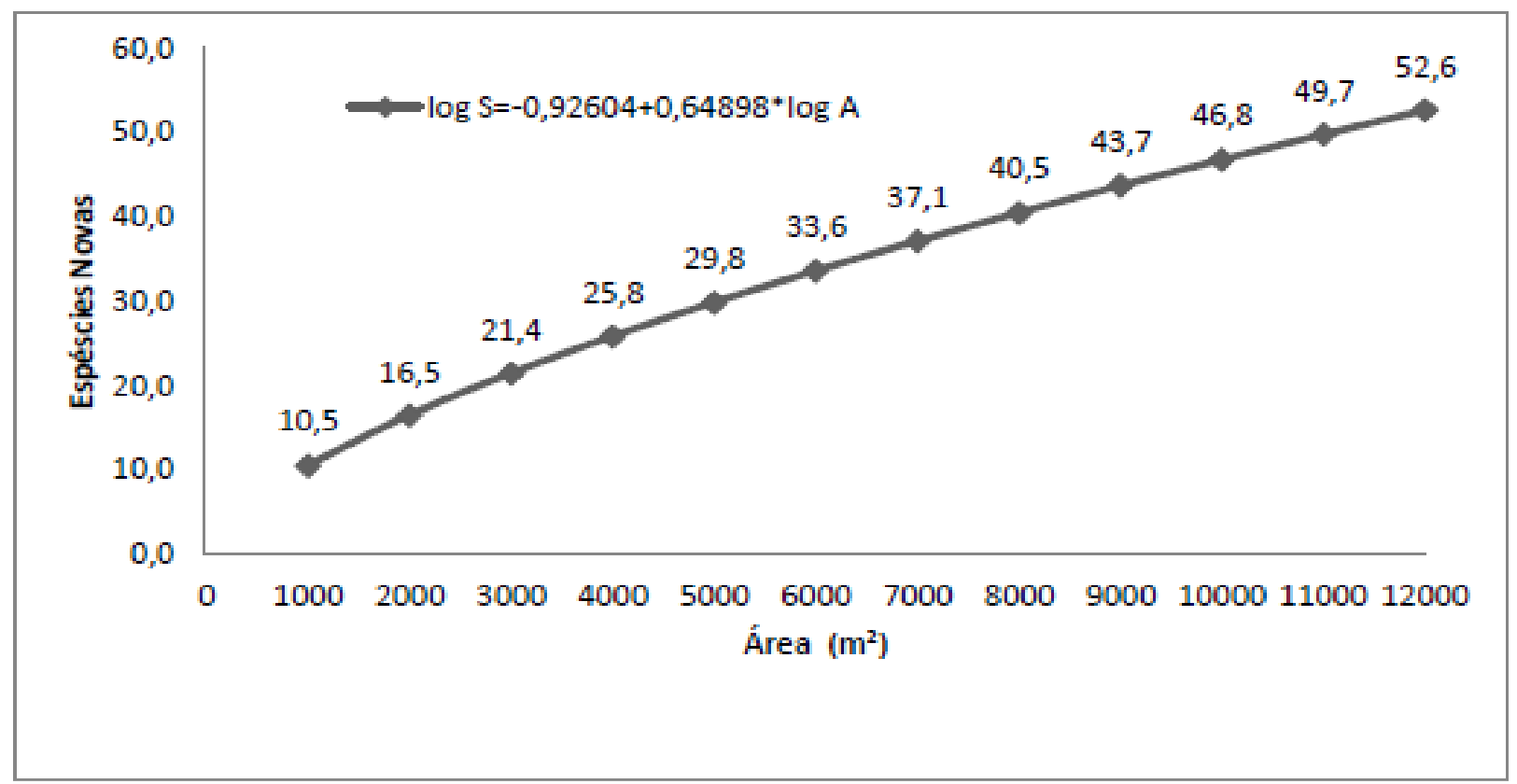

Fonte: Autores. 
Tabela 1 - Equações ajustadas e seus respectivos indicadores estatísticos de qualidade de ajuste (Teste F e sua respectiva probabilidade (p), Coeficiente de Determinação $\left(\mathrm{r}^{2} \%\right)$ e Coeficiente de Variação (CV \%)).

\begin{tabular}{lrrrr}
\hline \multicolumn{1}{c}{ Equações ajustadas } & $\boldsymbol{F}$ & $\boldsymbol{p}$ & $\boldsymbol{r}^{\mathbf{2}(\%)}$ & $\boldsymbol{C V}(\%)$ \\
\hline $\log \mathrm{S}=-0,92604+0,64898 * \log \mathrm{A}$ & 913,43 & 0,000 & 98,8 & 1,54 \\
$\mathrm{~S}=-117,79+17,714 * \ln \mathrm{A}$ & 134,15 & 0,000 & 92,4 & 11,25 \\
\hline
\end{tabular}

Fonte: Autores.

Este resultado não difere dos encontrados por Oliveira e Amaral (2004), em que a curva espécie-área, indicou que a comunidade vegetal estudada, 1ha de floresta na Amazônia Central, alcançou elevada diversidade de espécies em sua composição florística, denotando insuficiência amostral em seu estudo. Da mesma forma, Chilling \& Batista (2008) afirmam que em florestas tropicais é difícil a definição de limites para a amostragem, devido à alta riqueza de espécies, a curva apresenta estabilização com grandes tamanhos de amostra, pois sempre aparecerão novas espécies.

\section{Composição Florística}

De acordo com o levantamento foram registrados 320 indivíduos classificados em 29 famílias, 44 gêneros, 46 espécies e 4 morfoespécies, o equivalente a 41 espécies/ha. Do total de espécies, três foram identificadas até o nível de gênero e uma não foi determinada, pois a falta de material fértil dificultou a identificação (Tabela 2).

Tabela 2 - Lista das espécies, famílias, habito, grupo ecológico (GE) sendo: Pi- Pioneira; SI- Secundária inicial; STSecundária tardia; SC- Sem classificação) e classificações das espécies quanto ao hábito em: (Árvore, arbusto, erva, liana, palmeira e sem classificação), Paragominas-PA e número de indivíduos registrados nas unidades amostrais implantadas na área do fragmento florestal, no Platô Miltônia III da Mineração Paragominas S.A., Paragominas, PA.

\begin{tabular}{|c|c|c|c|c|}
\hline Espécies & Família & Habito & GE & Ind. \\
\hline Abarema jupunba (Willd.) Britton \& Killip & Fabaceae & Árvore & ST & 1 \\
\hline Apeiba glabra Aubl. & Malvaceae & Árvore & $\mathrm{SI}$ & 23 \\
\hline Amphiodon effusus Huber & Fabaceae & Árvore & SC & 3 \\
\hline Bellucia grossularioides (L.) Triana & Melastomataceae & Árvore & SI & 3 \\
\hline Byrsonima sp. & Malpighiaceae & Arbusto & SI & 12 \\
\hline Caryocar glabrum (Aubl.) Pers. & Caryocaraceae & Árvore & ST & 1 \\
\hline Chamaecrista xinguensis (Ducke) H.S. Irwin \& Barneby & Fabaceae & Árvore & $\mathrm{Pi}$ & 2 \\
\hline Cecropia sciadophylla Mart. & Urticaceae & Árvore & $\mathrm{Pi}$ & 28 \\
\hline Cenostigma tocantinum Ducke & Fabaceae & Árvore & $\mathrm{SI}$ & 1 \\
\hline Cordia scabrifolia A.DC. & Boraginaceae & Árvore & ST & 7 \\
\hline Dodecastigma integrifolium (Lanj.) Lanj. \& Sandwith & Euphorbiaceae & Árvore & $\mathrm{Pi}$ & 1 \\
\hline Ecclinusa guianensis Eyma & Sapotaceae & Árvore & ST & 1 \\
\hline Eschweilera coriacea (DC.) S.A. Mori & Lecythidaceae & Árvore & ST & 26 \\
\hline Euxylophora paraensis Huber & Rutaceae & Árvore & ST & 1 \\
\hline Goupia glabra Aubl. & Goupiaceae & Árvore & ST & 1 \\
\hline Guarea carinata Ducke & Meliaceae & Árvore & SC & 1 \\
\hline Guatteria poeppigiana Mart. & Annonaceae & Árvore & $\mathrm{Pi}$ & 13 \\
\hline Guatteria sp. & Annonaceae & Árvore & ST & 1 \\
\hline Hydrochorea sp. & Fabaceae & Árvore & SC & 3 \\
\hline Inga alba (Sw) Willd. & Fabaceae & Árvore & SI & 31 \\
\hline
\end{tabular}




\begin{tabular}{|c|c|c|c|c|}
\hline Inga thibaudiana DC. & Fabaceae & Árvore & $\mathrm{SI}$ & 13 \\
\hline Iryanthera juruensis Warb. & Myristicaceae & Árvore & ST & 3 \\
\hline Jacaranda copaia (Aubl.) D.Don & Bignoniaceae & Árvore & $\mathrm{Pi}$ & 14 \\
\hline Laetia procera (Poepp.) Eichler & Salicaceae & Árvore & $\mathrm{SI}$ & 14 \\
\hline Licania kunthiana Hook.f. & Chrysobalanaceae & Árvore & ST & 5 \\
\hline Ocotea opifera Mart. & Lauraceae & Árvore & $\mathrm{Pi}$ & 7 \\
\hline Oenocarpus bacaba Mart. & Arecaceae & Palmeira & $\mathrm{Pi}$ & 1 \\
\hline Parkia paraensis Ducke & Fabaceae & Árvore & ST & 1 \\
\hline Pouteria decorticans T.D. Penn. & Sapotaceae & Arbusto & ST & 13 \\
\hline Protium sp. & Burseraceae & Árvore & SC & 1 \\
\hline Protium tenuifolium (Engl.) Engl. & Burseraceae & Árvore & ST & 5 \\
\hline Pseudopiptadenia leptostachya (Benth.) Rauschert & Fabaceae & Árvore & ST & 1 \\
\hline Pterocarpus amazonum (Mart. ex Benth.) Amshoff & Fabaceae & Árvore & $\mathrm{SI}$ & 2 \\
\hline Rhabdodendron amazonicum (Spruce ex Benth.) & Rhabdondendraceae & Árvore & SC & 1 \\
\hline Rinorea flavescens (Aubl.) Kuntze & Violaceae & Árvore & $\mathrm{SI}$ & 28 \\
\hline Sapium marmieri Huber & Euphorbiaceae & Árvore & $\mathrm{Pi}$ & 1 \\
\hline Schefflera morototoni (Aubl.) Maguire, Steyerm. \& Frodin & Araliaceae & Árvore & $\mathrm{Pi}$ & 10 \\
\hline Simaba cedron Planch. & Simaroubaceae & Árvore & $\mathrm{SI}$ & 1 \\
\hline Spiranthera parviflora Sandwith & Rutaceae & Arbusto & $\mathrm{SI}$ & 1 \\
\hline Sterculia pruriens (Aubl.) K.Schum. & Malvaceae & Árvore & $\mathrm{SI}$ & 1 \\
\hline Tachigali guianensis (Benth.) Zarucchi \& Herend & Fabaceae & Árvore & SC & 1 \\
\hline Tapirira guianensis Aubl. & Anacardiaceae & Árvore & $\mathrm{Pi}$ & 4 \\
\hline Tetragastris altissima (Aulb.) Swart. & Burseraceae & Árvore & $\mathrm{SI}$ & 3 \\
\hline Trattinnickia peruviana Loes. & Burseraceae & Árvore & $\mathrm{Pi}$ & 1 \\
\hline Trichilia micrantha Benth. & Meliaceae & Árvore & ST & 2 \\
\hline Jacaranda copaia (Aubl.) D.Don & Hypericaceae & Árvore & $\mathrm{Pi}$ & 6 \\
\hline Wallacea insignis Spruce ex Benth. \& Hook.f. & Ochnaceae & Árvore & $\mathrm{Pi}$ & 4 \\
\hline Xylopia nitida Dunal & Annonaceae & Árvore & $\mathrm{SI}$ & 11 \\
\hline \multirow[t]{2}{*}{ Zanthoxylum rhoifolium Lam. } & Rutaceae & Árvore & $\mathrm{Pi}$ & 4 \\
\hline & & & & 319 \\
\hline
\end{tabular}

Fonte: Autores.

As oito famílias que apresentaram maior riqueza de espécies foram Fabaceae (22\%), seguida de Burseraceae (8\%), Rutaceae (6 \%), Annonaceae, Euphorbiaceae, Malvaceae, Meliaceae e Sapotaceae com (4 \%), as quais participaram com $56 \%$ da riqueza total.

As nove famílias botânicas com maior número de indivíduos foram Fabaceae $(18,43 \%)$, seguida de Urticaceae $(8,75$ $\%)$, Violaceae $(8,75 \%)$, Lecythidaceae (8,12 \%), Annonaceae (7,81 \%), Malvaceae (7,5 \%), Bignoniaceae, Salicaceae, Sapotaceae $(4,37 \%)$.

Ao levar em consideração tanto o número de indivíduos quanto o número de espécies a família mais importante foi Fabaceae.

Martins (1979) define que espécies raras são aquelas que apresentam apenas um indivíduo na amostragem. Para Nappo (1999) essas espécies são as grandes responsáveis pela alta diversidade nas florestas tropicais. Desta forma, 21 famílias (Anacardiaceae, Araliaceae, Arecaceae, Bignoniaceae, Boraginaceae, Caryocaraceae, Chrysobalanaceae, Goupiaceae, Hypericaceae, Lauraceae, Lecythidaceae, Malpighiaceae, Melastomataceae, Myristicaceae, Nyctaginaceae, Ochnaceae, 
Rhabdondendraceae, Salicaceae, Simaroubaceae, Urticaceae, Violaceae), foram representadas por apenas uma espécie. Elevada raridade, também, foi encontrada por Pinheiro et al., cujos os resultados apontaram 14 famílias representadas por uma única espécie. A raridade é considerada um padrão comum para a maioria das comunidades florestais (Hubbel e Foster, 1986; Huston, 1994)

Desse conjunto, a maior raridade ocorreu nas famílias Arecaceae, Caryocaraceae, Goupiaceae, Nyctaginaceae, Simaroubaceae, as quais tiveram suas espécies representadas por um único indivíduo.

Whitmore (1990), afirmou que Fabaceae, Annonaceae, Euphorbiaceae, Lauraceae, Moraceae, Myristicaceae, Rubiaceae e Sapotaceae são as mais representativas nas regiões tropicais. No presente estudo Fabaceae apresentou maior riqueza de espécies. De modo geral essa é a família que se destaca na maioria dos levantamentos florísticos realizados na Amazônia, tanto em florestas naturais primárias quanto nas florestas secundárias (Melo, 2004).

Mendes et al., (2012), verificaram altas frequências de Fabaceae na composição florística do sub-bosque de uma floresta de terra firme, na Amazônia Oriental, no município de Moju, Nordeste do Pará. O mesmo resultado foi encontrado por Francez et al., (2007) e Pinheiro et al., (2007), no município de Paragominas, Sudeste Paraense, em um fragmento de floresta primária. Pereira et al., (2011), encontrou 24 espécies dessa família em levantamento feito em arbóreas com DAP a partir de 10 cm, em floresta de Terra firme no Estado do Amapá. Esse comportamento também é confirmado por Miranda (2000), em Rondônia, que encontrou 47 espécies dessa família, em florestas de terra firme, matas ciliares e alagadas.

Burseraceae e Rutaceae aparecem entre as 5 mais representativas na área de estudo, por serem respectivamente a segunda e terceira famílias com maior riqueza de espécies neste estudo. Para Leitão Filho (1987), Burseraceae e Lecytidaceae, estão entre as famílias que apresentam maior número espécies e indivíduos em florestas tropicais de terra firme na Amazônia brasileira.

No estudo realizado por Mendes et al. (2013), em vegetação de sub-bosque de uma floresta de terra firme, no município de Moju, a maior riqueza de espécies foi apresentada por Burseraceae, o que confirma a posição de destaque apresentada por essa família nesse estudo. As características ambientais e o histórico de antropização de áreas são definidoras dos processos de sucessão florestal em florestas secundárias, e influenciaram na composição florística da área de estudo (Martins, et al. 2008).

\section{Estrutura Fitossociológica}

As espécies que apresentaram maior abundância foram: Inga alba (9,7 \%), Rinorea flavescens e Cecropia sciadophylla (8,75\%), Eschweilera coriacea (8,12\%), Apeiba glabra (7,18\%), Laetia procera e Jacaranda copaia (4,4 \%) (Tabela 3).

Tabela 3 - Parâmetros fitossociológicos calculados para a área do fragmento de floresta secundária, Paragominas, PA.(Ni = ${ }^{\circ}$ de indivíduos; $\mathrm{Aa}=$ abundância absoluta; $\mathrm{Ar}=$ abundância relativa; $\mathrm{Fa}=$ frequência absoluta; $\mathrm{Fr}=$ frequência relativa; Da $=$ Dominância absoluta; Dr = dominância relativa; IVI = índice de valor de importância; H' = índice de de Shannon.

\begin{tabular}{|c|c|c|c|c|c|c|c|c|}
\hline Espécies & $\mathbf{N i}$ & Aa & Ar & $\mathbf{F a}$ & Fr & Da & Dr & IVI \\
\hline Abarema jupunba (Willd.) Britton \& Killip & 1 & 0,83 & 0,31 & 0,08 & 0,62 & 0,01 & 0,13 & 1,07 \\
\hline Amphiodon effusus Huber & 3 & 2,50 & 0,94 & 0,17 & 1,24 & 0,08 & 0,71 & 2,89 \\
\hline Apeiba glabra Aubl. & 23 & 19,17 & 7,19 & 0,75 & 5,59 & 0,37 & 3,48 & 16,26 \\
\hline Bellucia grossularioides (L.) Triana & 3 & 2,50 & 0,94 & 0,17 & 1,24 & 0,08 & 0,78 & 2,96 \\
\hline
\end{tabular}


Byrsonima sp.

Caryocar glabrum (Aubl.) Pers.

Cassia xinguensis Ducke

Cecropia sciadophylla Mart.

Cenostigma tocantinum Ducke

Cordia scabrifolia A.DC.

Dodecastigma integrifolium (Lanj.) Lanj. \& Sandwith

Ecclinusa guianensis Eyma

Eschweilera coriacea (DC.) S.A. Mori

Euxylophora paraensis Huber

Goupia glabra Aubl.

Guarea carinata Ducke

Guatteria poeppigiana Mart.

Hydrochorea sp.

Indet.

Inga alba (Sw) Willd.

Inga thibaudiana DC.

Iryanthera juruensis Warb.

Jacaranda copaia (Aubl.) D.Don

Laetia procera (Poepp.) Eichler

Licania kunthiana Hook.f.

Neea cuspidata

Ocotea opifera Mart.

Oenocarpus bacaba Mart.

Parkia paraensis Ducke

Pouteria decorticans T.D. Penn.

Protium sp.

Protium tenuifolium (Engl.) Engl.

Pseudopiptadenia leptostachya (Benth.) Rauschert

Pterocapus amazonicus Huber

Rhabdodendron amazonicum (Spruce ex Benth.) Huber

Rinorea flavescens (Aubl.) Kuntz

Sapium marmieri Huber

Schefflera morototoni (Aubl.) Maguire

Simaba cedron Planch.

\begin{tabular}{|c|c|c|c|c|c|c|c|}
\hline 12 & 10,00 & 3,75 & 0,67 & 4,97 & 0,18 & 1,63 & 10,35 \\
\hline 1 & 0,83 & 0,31 & 0,08 & 0,62 & 0,01 & 0,09 & 1,02 \\
\hline 2 & 1,67 & 0,63 & 0,08 & 0,62 & 0,04 & 0,39 & 1,64 \\
\hline 28 & 23,33 & 8,75 & 0,83 & 6,21 & 1,03 & 9,57 & 24,54 \\
\hline 1 & 0,83 & 0,31 & 0,08 & 0,62 & 0,01 & 0,12 & 1,06 \\
\hline 7 & 5,83 & 2,19 & 0,33 & 2,48 & 0,13 & 1,20 & 5,87 \\
\hline 1 & 0,83 & 0,31 & 0,08 & 0,62 & 0,03 & 0,25 & 1,19 \\
\hline 1 & 0,83 & 0,31 & 0,08 & 0,62 & 0,01 & 0,08 & 1,02 \\
\hline 26 & 21,67 & 8,13 & 0,58 & 4,35 & 1,43 & 13,31 & 25,78 \\
\hline 1 & 0,83 & 0,31 & 0,08 & 0,62 & 0,29 & 2,67 & 3,60 \\
\hline 1 & 0,83 & 0,31 & 0,08 & 0,62 & 0,02 & 0,14 & 1,08 \\
\hline 1 & 0,83 & 0,31 & 0,08 & 0,62 & 0,07 & 0,65 & 1,58 \\
\hline 13 & 10,83 & 4,06 & 0,50 & 3,73 & 0,25 & 2,29 & 10,08 \\
\hline 3 & 2,50 & 0,94 & 0,08 & 0,62 & 0,05 & 0,42 & 1,98 \\
\hline 1 & 0,83 & 0,31 & 0,08 & 0,62 & 0,01 & 0,13 & 1,06 \\
\hline 31 & 25,83 & 9,69 & 0,92 & 6,83 & 0,71 & 6,63 & 23,15 \\
\hline 13 & 10,83 & 4,06 & 0,58 & 4,35 & 0,71 & 6,60 & 15,01 \\
\hline 3 & 2,50 & 0,94 & 0,25 & 1,86 & 0,08 & 0,74 & 3,54 \\
\hline 14 & 11,67 & 4,38 & 0,67 & 4,97 & 0,44 & 4,08 & 13,42 \\
\hline 14 & 11,67 & 4,38 & 0,58 & 4,35 & 0,20 & 1,82 & 10,54 \\
\hline 5 & 4,17 & 1,56 & 0,17 & 1,24 & 0,60 & 5,53 & 8,34 \\
\hline 1 & 0,83 & 0,31 & 0,08 & 0,62 & 0,01 & 0,09 & 1,02 \\
\hline 7 & 5,83 & 2,19 & 0,50 & 3,73 & 0,24 & 2,18 & 8,10 \\
\hline 1 & 0,83 & 0,31 & 0,08 & 0,62 & 0,04 & 0,37 & 1,31 \\
\hline 1 & 0,83 & 0,31 & 0,08 & 0,62 & 0,02 & 0,17 & 1,10 \\
\hline 13 & 10,83 & 4,06 & 0,50 & 3,73 & 0,70 & 6,53 & 14,32 \\
\hline 1 & 0,83 & 0,31 & 0,08 & 0,62 & 0,02 & 0,20 & 1,13 \\
\hline 5 & 4,17 & 1,56 & 0,33 & 2,48 & 0,24 & 2,23 & 6,28 \\
\hline 1 & 0,83 & 0,31 & 0,08 & 0,62 & 0,04 & 0,39 & 1,33 \\
\hline 2 & 1,67 & 0,63 & 0,17 & 1,24 & 0,19 & 1,73 & 3,59 \\
\hline 1 & 0,83 & 0,31 & 0,08 & 0,62 & 0,03 & 0,25 & 1,19 \\
\hline 28 & 23,33 & 8,75 & 0,50 & 3,73 & 0,83 & 7,72 & 20,20 \\
\hline 1 & 0,83 & 0,31 & 0,08 & 0,62 & 0,01 & 0,09 & 1,02 \\
\hline 10 & 8,33 & 3,13 & 0,42 & 3,11 & 0,47 & 4,40 & 10,63 \\
\hline 1 & 0,83 & 0,31 & 0,08 & 0,62 & 0,01 & 0,10 & 1,03 \\
\hline
\end{tabular}


Spiranthera parviflora Sandwith

Sterculia pruriens (Aubl.) K.Schum.

Tachigali guianensis (Benth.) Zarucchi \& Herend

Tapirira guianensis Aubl.

Tetragastris altissima (Aulb.) Swart.

Trattinnickia peruviana Loes.

Trichilia micrantha Benth.

Vismia guianensis (Aubl.) Pers

Wallacea insignis Spruce ex Benth. \& Hook.f.

Xylopia nitida Dunal

Zanthoxyllon rhoifolium Lam.

\begin{tabular}{llllllll}
1 & 0,83 & 0,31 & 0,08 & 0,62 & 0,04 & 0,39 & 1,33 \\
1 & 0,83 & 0,31 & 0,08 & 0,62 & 0,02 & 0,19 & 1,13 \\
1 & 0,83 & 0,31 & 0,08 & 0,62 & 0,01 & 0,13 & 1,07 \\
4 & 3,33 & 1,25 & 0,25 & 1,86 & 0,19 & 1,74 & 4,86 \\
3 & 2,50 & 0,94 & 0,25 & 1,86 & 0,07 & 0,63 & 3,43 \\
1 & 0,83 & 0,31 & 0,08 & 0,62 & 0,02 & 0,18 & 1,11 \\
2 & 1,67 & 0,63 & 0,17 & 1,24 & 0,16 & 1,49 & 3,36 \\
6 & 5,00 & 1,88 & 0,33 & 2,48 & 0,14 & 1,33 & 5,69 \\
4 & 3,33 & 1,25 & 0,25 & 1,86 & 0,08 & 0,79 & 3,90 \\
11 & 9,17 & 3,44 & 0,42 & 3,11 & 0,26 & 2,44 & 8,98 \\
4 & 3,33 & 1,25 & 0,25 & 1,86 & 0,09 & 0,81 & 3,92 \\
\hline 320 & 266,67 & 100,00 & 13,42 & 100,00 & 10,78 & 100,00 & 300,00 \\
\hline
\end{tabular}

Fonte: Autores.

Esse resultado corrobora parcialmente com o encontrado por Francez et al. (2007), também em Paragominas, em Floresta ombrófila densa primária, que ao coletar dados, em duas ocasiões (2003, antes da exploração de madeira e em 2004, após a exploração madeireira) em 36 parcelas permanentes quadradas de 0,25 ha, sendo doze para estudar a floresta não explorada, doze para a explorada, com todos os indivíduos com DAP $>10 \mathrm{~cm}$ registrou a maior abundância de indivíduos para Rinorea flavescens, seguida de Lecythis idatimon, Poecilanthe effusa, Eschweilera grandiflora, Eschweilera pedicellata e Inga $s p$. Sandel e Carvalho (2000), ao estudarem a composição florística e a estrutura em uma área de floresta localizada na FLONA Tapajós (Oeste do Pará), verificaram que Rinorea flavescens, também, apresentou maior abundância, com 12,9 \% em relação às demais espécies. Já no estudo de Lima et al. (2012), em floresta ombrófila densa, no Estado do Amazonas, foi evidenciado que as três espécies com maior densidade foram: Eschweilera odora, Pouteria guianensis e Inga sp.

Dentre as espécies dominantes neste estudo destacam-se: Eschweilera coriacea (13,31\%), Cecropia sciadophylla $(9,57 \%)$, Rinorea flavescens (7,72 \%), Inga alba (6,63 \%), Inga thibaudiana (6,6\%), Pouteria decorticans (6,53 \%), Protium tenuifolium $(5,53 \%)$.

Resultados encontrados por Pinheiro et al. (2007), em floresta ombrófila densa, em Paragominas, determinaram que as espécies com maiores valores de dominância foram: Pouteria heterosepala, Eschweilera blanchetiana, Eschweilera amazonica, Manilkara paraensis, Protium apiculatum, Micropholis venulosa, Inga breviala. Da mesma forma, Lima et al. (2012) em levantamento florístico realizado em floresta ombrófila densa, no Estado do Amazonas, verificou que as espécies com maior dominância foram: Licania oblongiflora, Eschweilera odora, Pouteria guianensis, Xylopia nitida, Copaifera multijuga, Inga sp., Apuleia molaris. Assim os estudos denotam que em relação a abundância e dominância das espécies nas áreas estudadas não existe o predomínio de uma única espécie. Inga alba e Eschweilera coriacea por exemplo, foram as espécies mais abundantes e dominantes neste estudo, mas não predominaram e nenhum dos levantamentos florísticos realizados em floresta ombrófila densa. Isto confirma a afirmativa de Richards et al. (1996) de que em cada localidade há um conjunto característico de espécies que se destacam.

É importante frisar que Inga alba ocupou a primeira posição em termos de abundância na área. De acordo com Mata e Felix (2007), esse gênero é importante na recuperação de áreas degradadas, apresenta importante função ecológica no ecossistema, além do seu fruto servir de alimento para a fauna silvestre. 
Francez et al. (2013) afirmou que Rinorea flavescens, é muito abundante e frequente em florestas nativas no Estado do Pará, contudo pouco dominante. Essas informações também foram constatadas parcialmente no presente levantamento, onde Rinorea flavescens foi a segunda espécie mais abundante, porém não está entre as dez espécies mais frequentes, e é classificada como a terceira espécie dominante, e apresenta $7,72 \%$, do total da dominância entre as espécies levantadas (Tabela 3).

A análise do valor de importância das espécies, mostra que as dez espécies mais importantes foram: Eschweilera coriacea (8,59 \%), Cecropia sciadophylla (8,18 \%), Inga alba (7,2 \%), Rinorea flavescens (6,73\%), Apeiba glabra $(4,95)$, Inga thibaudiana (5\%), conforme tabela 3. Essas espécies não são qualificadas como de interesse comercial. Para Pinheiro et al. (2007), espécies que não apresentem valor comercial elevado, mas que apresentem elevado valor de importância podem está indicando que desempenham funções chaves em seus ecossistemas.

\section{Diversidade de espécies}

Neste estudo o índice de diversidade de Shannon-Weaner foi de 3,29. De acordo com Knight (1975) os valores de diversidade em florestas tropicais variam de 3,83 a 5,85. A diversidade de espécies neste estudo é considerada baixa, se comparado com o índice Shannon-Weaner encontrado em Paragominas por Francez (2007), antes da exploração de 4,29 e de 4,27 depois da exploração na área. Em levantamento em floresta secundária nos municípios de Marituba e Bragança, mesma mesorregião deste estudo, foram feitas medições nas espécies arbóreas com DAP $\geq 5 \mathrm{~cm}$, em 25 parcelas de 50 x $50 \mathrm{~m}$ subdivididas em subparcelas de $10 \mathrm{~m}$ x $10 \mathrm{~m}$. Para essas parcelas os índices de Shannon-Weaner foram encontrados os valores, respectivamente de 4,42 e 4,08. A baixa diversidade de espécies encontrada no presente estudo pode ser explicada pelo alto grau de antropismo na área, pois localiza-se em uma área no entorno de uma exploração de bauxita, além de que índices de diversidade baixos são comuns em florestas secundárias, devido à seletividade do ambiente, que exige alta capacidade adaptativa das espécies que nele se instalem inicialmente. Almeida, et al. (2015), encontraram em uma área de remanescentes de floresta estacional decidual para o grupo de espécies com $\mathrm{CAP} \geq 30 \mathrm{~cm}$, uma diversidade de espécies, medida pelo índice de Shannon (H') de 3,12 sendo considerado pelos autores de diversidade moderada para aquele tipo de floresta. Longhi et al. (1999), também encontraram para a área de um fragmento florestal, do mesmo tipo florestal uma diversidade de 3,21, valores próximos ao encontrado no presente estudo, este fragmento de floresta tropical antropizada.

Lima et al. (2012), citam que existem grandes dificuldades nas comparações entre os resultados de diferentes estudos quantitativos, pois são utilizadas uma grande variação de metodologias, as quais diferem no tamanho, número de parcelas e faixa de mensuração de DAP.

\section{Grupos ecológicos}

$\mathrm{Na}$ classificação das espécies em grupos ecológicos, pioneiras e secundárias tardias, estas contribuíram com os mesmos valores de espécies em cada grupo, perfazendo juntas $60 \%$, Figura 2. As espécies secundárias iniciais também contribuíram com elevado valor $28 \%$, evidenciando que a floresta se encontra em avançado estado de sucessão ecológica (Colonetti et al., 2009). A presença de $30 \%$ de espécies pioneiras pode ser explicada pela exploração madeireira ocorrida no local, a qual pode ter provocado abertura de clareiras e consequentemente maior entrada de luz na floresta, fator que pode ter estimulado o número elevado de germinação e estabelecimento desse grupo de espécies. 
Figura 2 - A: Classificação das espécies em grupos ecológicos (PI- Pioneira; SI- Secundária inicial; ST- Secundária tardia; SC- Sem classificação) no fragmento florestal, em, Paragominas, PA; B: Classificação das espécies quanto ao hábito em árvore, arbusto, erva, liana, palmeira e sem classificação no fragmento florestal, Paragominas, PA.

$\mathbf{A}$

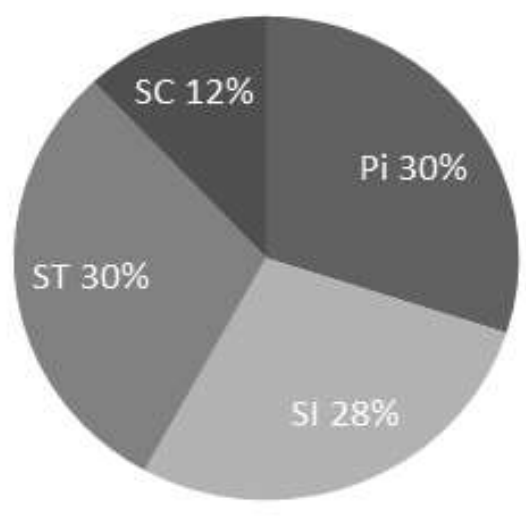

PI $\quad$ SI $\quad$ ST $\quad$ SC
B

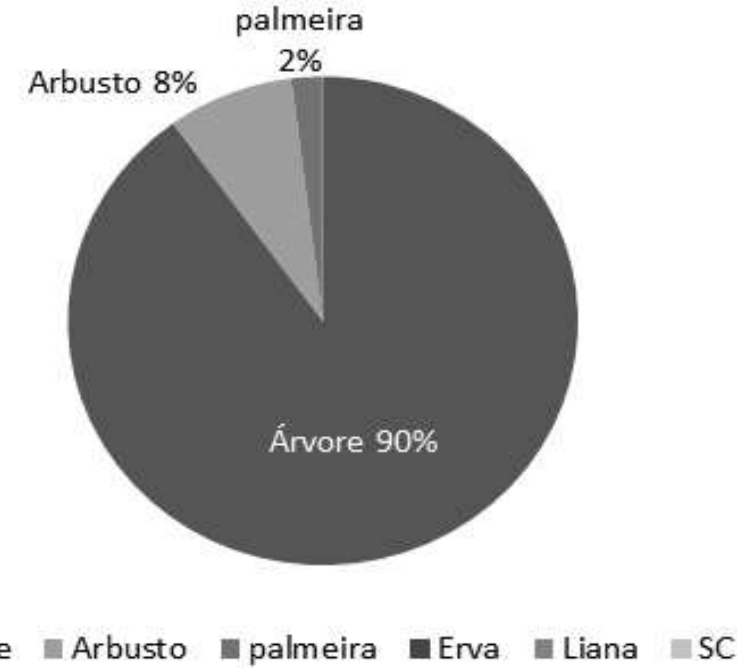

Fonte: Autores.

As espécies pioneiras são importantes cicatrizadoras das áreas degradadas, por isso são fundamentais nas atividades de recuperação, agindo como facilitadoras das espécies dos grupos mais avançados de sucessão (Batista Neto, 2007). Bem como, fornecem a rápida cobertura de áreas desnudas, devido ao crescimento rápido de suas espécies, ajudando no controle da erosão (Carter E Ungar, 2002).

No fragmento florestal $90 \%$ das espécies são árvores, $8 \%$ arbustos, e $2 \%$ palmeiras. O predomínio de determinadas formas de vidas depende das características ambientais da área, do tipo de pressão sofrida e também do que ocorreu no seu entorno (Araújo et al., 2005). Neste caso, o predomínio das arbóreas evidencia o elevado avanço da sucessão florestal na área de estudo.

\section{Conclusão}

A avaliação da estrutura fitossociológica realizado no fragmento florestal apresentou diversidade de espécies baixa, considerando as características das florestas primárias da Amazônia e quando comparado com estudos realizados, em Paragominas.

A família botânica Fabaceae é a mais importante na área do fragmento, tanto pela ocorrência de indivíduos quanto o número de espécies.

A área apresentou alta riqueza de espécies pioneiras, secundárias iniciais e secundárias tardia, evidenciando elevado estágio de sucessão florestal, o caracterizando como fragmento de floresta antropizada.

\section{Referências}

Almeida, C. M. De, Araújo, M. M., Longhi, S. J., Rovedder, A.P., Sccoti, M. S. V., D’avila, M., Aimi, S. C., \& Tonetto, T. Da S. (2015). Análise de agrupamentos em remanescente de floresta estacional decidual. Ciência Florestal, 25, 781-789.

Araújo, F. S., Martins, S. V., Neto, J. A. A. M., Lani, J. L., \& Pires, I. E. (2005). Florística da vegetação arbustivo-arbórea colonizadora de uma área degradada por mineração de caulim, em Brás Pires, MG. Revista Árvore, 29, 983-992. 
Barros, P. L. C. de. (1986). Estudo fitossociológico de uma floresta tropical úmida no planalto de Curuá-Una, Amazônia brasileira. 158 p. Tese (Doutorado em Ciências Florestais) - Universidade Federal do Paraná, Curitiba.

Bastos, T. X., Pacheco, N. A., Figueirêdo, R. D. O., \& Silva, G. (2005). Características agroclimáticas do município de Paragominas. Embrapa Amazônia Oriental-Documentos (INFOTECA-E).

Batista Neto, J. P. B. (2007). Banco de sementes do solo de uma Floresta Estacional Semidecidual, em Viçosa, Minas Gerais. Ciência Florestal, Santa Maria, $17,311-320$.

Brower, J. E. \& Zar, J. H. (1977). Field and laboratory methods for general ecology. Wm. C. Brown Company - 194 p.

Carter, C. T, \& Ungar, L. A. (2002). Abovegroud vegetation, seed bank and soil analysis of a 31 -year-old forest restoration on coal mine spoil in southeastern Ohio. The American Midland Naturalist, Notre Dame, 147, 44-59.

Coelho, R. De F. R., Zarin, D. J., Miranda, I. S., \& Tucker, J. M. (2003). Análise florística e estrutural de uma floresta em diferentes estágios sucessionais no município de Castanhal, Pará. Acta Amazônica, vol.33, n.4, p. 563-582.

Colonetti, S., Citadini-Zanette, V., Martins, R., Dos Santos, R., Rocha, E., \& Jarenkow, J. A. (2009). Florística e estrutura fitossociológica em floresta ombrófila densa submontana na barragem do rio São Bento, Siderópolis, Estado de Santa Catarina. Acta Scientiarum. Biological Sciences, $31,397-405$.

Chaves, A. D. C. G., Santos, R. M. S., Santos, J. O., Fernandes, A. A., \& Maracajá, P. B. (2013). A importância dos levantamentos florísticos e fitossociológicos para a conservação e preservação das florestas. ACSA - Agropecuária Científica no Semiárido, 9, 43-48

De Andrade Lima, R. B., Da Silva, J. A. A., Marangon, L. C., Ferreira, R. L. C., \& Da Silva, R. K. S. (2012). Fitossociologia de um trecho de floresta ombrófila densa na Reserva de Desenvolvimento Sustentável Uacari, Carauari, Amazonas. Scientia Plena, v. 8, n. 1.

Felfili, J. M., \& Rezende, R. P. (2003). Conceitos e métodos em fitossociologia. Ed. UNB. 68 p. Comunicações técnicas florestais, 5.

Finol, U. H. (1975). La silvicultura em la Orinoquia Venezolana. Revista forestal venezolana, 18, 37-114.

Flora do Brasil. (2020) em construção. Jardim Botânico do Rio de Janeiro. Disponível em: http://floradobrasil.jbrj.gov.br/.

Francez, L.M. De B, Carvalho, J. O. P De, Batista, F. De J, Jardim, F.C. Da S., \& Ramos, E. M. L. S. (2013). Influência da exploração florestal de impacto reduzido sobre as fases de desenvolvimento de uma floresta de terra firme, Pará, Brasil, Ciência Florestal, 23, 743-753.

Francez, L.M. De B, Carvalho, J. O. P De, Jardim, F.C. Da S., \& Ramos, E. M. L. S. (2007). Mudanças ocorridas na composição florística em decorrência da exploração florestal em uma área de floresta de terra firme na região de Paragominas, PA, Acta Amazônica, 37, $219-228$.

Gandolfi, S., Leitão Filho, H. F., \& Bezerra, C. L. E. (1995). Levantamento florístico e caráter sucessional das espécies arbustivo arbóreas de uma floresta mesófila semidecídua no município de Guarulhos, SP. Revista Brasileira de Biologia, 55, 753-767.

Hack, C., Longhi, S. J., Boligon, A. A., Murari, A. B., \& Pauleski, D. T. (2005). Análise fitossociológica de um fragmento de floresta estacional decidual no município de Jaguari, RS. Ciência Rural, 35, 1083-1091.

Hosokawa, T.R., Moura, De J. B., \& Cunha, U. S. (2008). Introdução ao Manejo e economia de florestas. UFPR.

Hubbell, S. P., \& Foster, R. B. (1986). Commonness and rarity in a neotropical forest: implications for tropical tree conservation.

Huston, M. A. (1994). Biological diversity: the coexistence of species on changing landscapes. Cambridge University Press. 681 p.

Knight, D. H. (1975). A phytosociological analysis of species-rich tropical forest on Barro Colorado Island, Panama. Ecological monographs, $45,259-284$.

Lamprecht, H. (1964). Ensayo sobre la estrutura floristica de la parte sur-oriental del Bosque Universitário: "El caimital", Estado Barinas. Revista forestal venezolana, 7, 77-119.

Leitão Filho, H. De F. (1987). Considerações sobre a florística de florestas tropicais e sub-tropicais do Brasil. IPEF, 35, 41-46.

Lima, R. B. A., Da Silva, J. A. A., Marangon, L. C., Ferreira, R. L. C., \& Silva, R. K. S. (2012). Fitossociologia de um trecho de floresta ombrófila densa na Reserva de Desenvolvimento Sustentável Uacari, Carauari, Amazonas. Scientia Plena, v. 8, n. 1.

Longhi, S. J., Nascimento, A. R. T., Fleig, F. D., Della-Flora, J. B., Freitas, R. A., \& Charão, L. W. (1999). Composição florística e estrutura da comunidade arbórea de um fragmento florestal no município de Santa Maria-Brasil. Ciência Florestal, 9, 115-133.

Longhi, S. J., Nascimento, A. R. T., Fleig, F. D., Della-Flora, J. B., Freitas, R. A. D., \& Charão, L. W. (1999). Composição florística e estrutura da comunidade arbórea de um fragmento florestal no município de Santa Maria, Brasil. Ciência Florestal, 9, n115-133.

Martins, F. R. (1979). O método de quadrantes ea fitossociologia de uma floresta residual do interior do Estado de Sao Paulo: Parque Estadual de Vassununga. USP.

Martins, S. V. (2012). Restauração ecológica de Ecossistemas degradados. Ed. UFV. 293p.

Mata, M. F., \& Felix, L. P. (2007). Flora da Paraíba, Brasil: Inga Mill (Leguminosae-mimosoideae). Revista Brasileira de Biociências, 5 (2): $135-137$.

Melo, M. S. (2004). Florística, fitossociologia e dinâmica de duas florestas secundárias antigas com histórias de uso diferentes no nordeste do Pará-Brasil. 116 f. Dissertação (Recursos Florestais) - Escola Superior de Agricultura, "Luiz de Queiroz", São Paulo. 
Mendes, F. S., Jardim, F. C. S., Carvalho, J. O. P., Lima, T. T. S., \& Souza, D. V. (2012). Dinâmica da composição florística do sub-bosque em floresta tropical manejada, no município de Moju, estado do Pará, Brasil. Revista de Ciências Agrárias Amazonian Journal of Agricultural and Environmental Sciences, 55, 117-123.

Mendes, F. D. S., Jardim, F. C. D. S., Carvalho, J. O. P. D., Souza, D. V., Araújo, C. B., Oliveira, M. G. D., \& Leal, E. D. S. (2013). Dinâmica da estrutura da vegetação do sub-bosque sob influência da exploração em uma floresta de terra firme no município de Moju-PA. Ciência Florestal, 23 , n. 2, p. $377-389$.

Miranda, I. S. (2000). Análise florística e estrutural da vegetação lenhosa do Rio Comemoração Pimenta Bueno, Rondônia, Brasil, Acta Botânica, 30, 393-422.

Moro, M. F., \& Martins, F. R. (2011). Métodos de levantamento do componente arbório-arbustivo. In: Felfili, J. M. et, al Fitossociologia no Brasil: Métodos e estudos de casos. Ed. UFV.

Nappo, M. E. (1999). Inventário florístico e estrutural da regeneração natural no sub-bosque de povoamentos homogêneos de Mimosa scabrella Bentham, implantados em áreas mineradas, em Poços de Caldas, Minas Gerais. 87 p. Dissertação (Mestrado em Engenharia Florestal) - Universidade Federal de Lavras, Lavras.

Oliveira, A. N. De, \& Amaral, I. L. Do. (2004). Florística e fitossociologia de uma floresta de vertente na Amazônia Central, Amazonas, Brasil. Acta Amazonica, 34, 21-34.

Pereira, L. A., Sobrinho, F. A. P., \& Da Costa Neto, S. V. (2011). Florística e estrutura de uma mata de terra firme na Reserva de Desenvolvimento Sustentável Rio Iratapuru, Amapá, Amazônia Oriental, Brasil. Floresta, 41

Pinheiro, K. A. O., Carvalho, J. C. P De, Quanz, B., Francez, L. M. De B., \& Schwartz. (2007). Fitossociologia de uma área de preservação permanente no leste da Amazônia: indicação de espécies para recuperação de áreas alteradas. Floresta, 37, 175-187.

Ribeiro, J. E. L. S., Hopkins, M. J. G., Vicentini, A., Sothers, C. A. Costa, M. A. Da S, Brito, J. M. De, De Souza, M. A. D., Martins, L. H. P., Lohmann, L. G., Assunção, P. A. C. L., Pereira, E. Da C., Silva, C. F. Da, Mesquita, M. R., \& Procópio, L. C. (1999). Flora da reserva Ducke: guia de identificação das plantas vasculares de uma floresta de terra-firme na Amazônia. INPA. 816p. il.

Richards, P. W., Frankham, R., \& Walsh, R. P. D. (1996). The tropical rain forest: an ecological study. Cambridge university press

Sandel, M. P., \& Carvalho, J. O. P. (2000). Composição florística e estrutura de uma área de cinco hectares de mata alta sem babaçu na Floresta Nacional do Tapajós. Embrapa Amazônia Oriental-Documentos (INFOTECA-E).

Schilling, A. C., \& Batista, J. L. Ferreira. (2008). Curva de acumulação de espécies e suficiência amostral em florestas tropicais. Brazilian Journal of Botany, v31, 179-187.

SER. (2004). Society for Ecologial Restoration, Princípios da SER International sobre a restauração ecológica. http://www.ser.org.

Souza, A. L., \& Soares, C. P. B. (2013). Florestas Nativas: estrutura, dinâmica e manejo. Ed. UFV.

Whitmore, T. C. (1990). An introduction to tropical rain forest. Clarendon Press, Oxford. 226pp. 